\title{
Vorticity banding in rodlike virus suspensions
}

\author{
Kyongok Kang, ${ }^{1}$ M. P. Lettinga, ${ }^{1}$ Z. Dogic, ${ }^{2}$ and Jan K. G. Dhont ${ }^{1}$ \\ ${ }^{1}$ Institute für Festkörper Forschung (IFF), Weiche Materie, Forschungszentrum Jülich, D52425 Jülich, Germany \\ ${ }^{2}$ Rowland Institute at Harvard, 100 Edwin H. Land Boulevard, Cambridge, Massachusetts 02142, USA
}

(Received 16 February 2006; published 28 August 2006)

\begin{abstract}
Vorticity banding under steady shear flow is observed in a suspension of semiflexible colloidal rods (fd virus particles) within a part of the paranematic-nematic biphasic region. Banding occurs uniformly throughout the cell gap within a shear-rate interval $\left(\dot{\gamma}_{-}, \dot{\gamma}_{+}\right)$, which depends on the fd concentration. For shear rates below the lower-border shear rate $\dot{\gamma}_{-}$only shear elongation of inhomogeneities, which are formed due to paranematicnematic phase separation, is observed. Within a small region just above the upper-border shear rate $\dot{\gamma}_{+}$, banding occurs heterogeneously. An essential difference in the kinetics of vorticity banding is observed, depending on the morphology of inhomogeneities formed during the initial stages of the paranematic-nematic phase separation. Particle tracking and polarization experiments indicate that the vorticity bands are in a weak rolling flow, superimposed on the applied shear flow. We propose a mechanism for the origin of the banding instability and the transient stability of the banded states. This mechanism is related to the normal stresses generated by inhomogeneities formed due to the underlying paranematic-nematic phase transition.
\end{abstract}

DOI: 10.1103/PhysRevE.74.026307

PACS number(s): 47.20.Ft, 47.55.-t, 47.15.Fe, 82.70.-y

\section{INTRODUCTION}

At equilibrium, complex fluids undergo a variety of ordering transitions that are driven by purely thermodynamic forces (see, for example, Refs. [1,2]). External fields can greatly affect the phase behavior of these systems. For nonconservative fields such as a shear flow no thermodynamic analog is yet known, where the equality of scalar quantities in coexisting phases (the analogs to pressure and chemical potential) suffices to predict the location of phase transition lines as a function of the strength of the driving force. The search for such a formalism remains at the forefront of research in soft condensed matter and nonequilibrium physics $[3,4]$. In addition to nonequilibrium phases, systems driven by a shear flow frequently exhibit hydrodynamically driven pattern formation which have no equilibrium analogs. Some representative examples include Taylor-Couette flow at high shear rates and shear banding at much lower shear rates [5-7].

A particularly important example of a complex fluid whose phase behavior is greatly affected by a shear flow is a system of rodlike colloids. At equilibrium rods undergo a thermodynamically driven phase transition from an isotropic to a liquid crystalline nematic phase [2]. Shear flow strongly aligns rods and therefore affects the location of the isotropic (paranematic-) nematic phase transition [8-10]. An isotropic state under shear flow is referred to as a "paranematic" state to indicate that flow partially aligns otherwise isotropic rods. The paranematic-nematic binodal is defined as the locus of points that separates the one-phase region from the region where phase coexistence occurs. The spinodal under shear flow is defined as the locus of points where the system becomes unstable against infinitesimally small perturbations of the orientational order parameter. The spinodal and binodal referred to here and hereafter in the present paper are connected to the paranematic-nematic phase transition, that is, the shear-affected isotropic-nematic phase transition that also occurs in the absence of flow. Whenever a spinodal or bin- odal is mentioned in this paper, it refers to the shear-affected isotropic-nematic phase transition and not to the banding transition, except when explicitly mentioned otherwise.

Besides shifts of the equilibrium binodals and spinodals, shear flow can also lead to the formation of banded structures in a number of complex fluids including rodlike colloids studied here. In general two types of banding transitions can be distinguished: gradient banding and vorticity banding. In the case of gradient banding, coexisting regions ("bands") extend along the gradient direction. The shear rate is essentially constant within these bands (see Refs. [11-16]). The gradient banding transition is relatively well understood and it occurs when the shear stress decreases with increasing shear rate [17-21]. In case of vorticity banding, regions of different internal structure are alternately stacked along the vorticity direction $[9,10,22-24]$. The origin and the mechanism of the vorticity banding transition are not yet known. It was suggested in Ref. [17] that vorticity banding can occur when the shear stress is a multivalued function of the shear rate. As far as we know, there are no systematic experimental studies of vorticity banding concerning shear-band formation kinetics, the characteristic features of vorticity-banded structures, the internal structure of individual bands, and the possible connection to the underlying nonequilibrium phase behavior.

In the present paper we systematically study pattern formation (vorticity-banding transition) under steady shear flow conditions of a suspension of rodlike colloids in shear flow, where bands of different orientational order are stacked along the vorticity direction. We quantify the relationship between pattern formation and the underlying nonequilibrium, shear-affected paranematic-nematic phase transition. Vorticity banding is observed within a part of the biphasic isotropic-nematic region, under both controlled shear-rate and shear-stress conditions. We propose a possible mechanism that describes the vorticity-banding instability and also explains the temporary stability of the quasistationary banded states. The proposed mechanism implies that there is no genuine stationary vorticity-banded state, and that its tran- 
sient stability relies on the presence of inhomogeneities formed due to paranematic-nematic phase separation. The lifetime of the vorticity-banded state is thus set by the lifetime of the inhomogeneities. As soon as the inhomogeneities disappear, for example due to sedimentation, the vorticitybanded state also disappears. This process takes a few days as compared to the formation of bands within an hour. Since vorticity banding occurs only inside the biphasic paranematic-nematic region, the inhomogeneities that form after a shear-rate quench due to paranematic-nematic phase separation seem to play a crucial role in rendering the system unstable against vorticity banding. The kinetics of the banding transition is found to be fundamentally different depending on whether these inhomogeneities are isolated or form an interconnected structure. Furthermore, above the region where bands are formed homogeneously, there is a small shear-rate range where heterogenous banding is observed.

As an experimental model system we use the monodisperse rodlike virus fd. Extensive experiments have shown that the equilibrium isotropic-nematic phase transition of $\mathrm{fd}$ virus is quantitatively described by Onsager's theory [25] when it is extended to take into account fd flexibility and its surface charge [26-29]. Besides fd, numerous other systems of rodlike particles exhibit an isotropic-nematic phase transition, with important examples including DNA [30], tobacco mosaic virus [31] and synthetic Boehmite rods [32]. The phase behavior of fd has been studied in the presence of an external magnetic (conservative) field in Ref. [33]. The induced shift of isotropic-nematic binodals and spinodals for such a conservative external field can be defined thermodynamically, simply by adding the corresponding potential energy to the Hamiltonian. The analogous procedure is not allowed for the shear flow due to its nonconservative nature.

Besides previous experiments on fd $[9,10]$, the only other experimental study of the paranematic-nematic phase transition of colloidal rods in shear flow, that we are aware of, is in dispersions of hydroxypropylcellulose [15]. However, there is significant work done on shear banding and phase transitions of related systems such as wormlike micelles $[11,12,23,34]$ and thermotropic liquid crystals [13,14]. A complication of wormlike micelles systems, when compared to suspensions of rods, is that the worm-length distribution and the scission and recombination kinetics depend on the shear rate.

The main body of this paper is organized as follows. In Sec. II, details of the fd virus suspensions, the experimental setup, and the data analysis are given. The topology of the shear-induced nonequilibrium phase diagram is given in Sec. III. The kinetics of band formation is described in Sec. IV. In Sec. V we describe experiments which indicate that the bands are in rolling flow. Finally, we propose a mechanism for the vorticity-banding instability in Sec. VI. This mechanism is reminiscent of the well-known elastic instability of polymers [35-39], where nonuniform elastic deformation of the polymers leads to a rolling flow. In the present case, elastic deformations of inhomogeneities formed due to paranematic-nematic phase separation lead to the vorticitybanding transition and the associated rolling flow.

\section{EXPERIMENTAL DETAILS}

In this section we discus the colloidal system and the experimental setup together with the data analysis.

\section{A. The colloidal system}

We use monodisperse rodlike fd viruses which are a good model system for studies of liquid crystalline phase behavior [26-29]. The bacteriophage fd is a semiflexible filamentous molecule with a contour length $L=880 \mathrm{~nm}$ and a diameter $D=6.6 \mathrm{~nm}$. The persistence length is $P=2200 \mathrm{~nm}$, which is more than twice its contour length. Fd virus is thus relatively stiff. The molecular weight of native fd is $1.64 \times 10^{7} \mathrm{~g} / \mathrm{mol}$. Bacteriophage fd was grown and purified following standard biological protocols, using the Xl1-blue strain of Escherichia coli as the host bacteria [40]. The standard yield was approximatively $15 \mathrm{mg}$ of fd per liter of infected bacterial culture. The virus particles were purified by repeated centrifugation $\left(10^{5} \mathrm{~g}\right.$ for $\left.5-6 \mathrm{~h}\right)$, and redispersed in high-ionicstrength buffer to screen electrostatic interactions $(20 \mathrm{mM}$ tris- $\mathrm{HCl}, p \mathrm{H} 8.15,100 \mathrm{mM} \mathrm{NaCl})$. Dextran $(507 \mathrm{kD}$, radius of gyration $16 \mathrm{~nm}$, Sigma-Aldrich) was mixed with the $\mathrm{fd}$ dispersion in order to widen the biphasic region and enhance the phase-separation kinetics [29].

Two different dispersions are used in the experiments with two different dextran concentrations. To study the nonequilibrium kinetics and phase diagram we used a low dextran concentration $(10.6 \mathrm{mg} / \mathrm{ml}$ dextran, $21.7 \mathrm{mg} / \mathrm{ml} \mathrm{fd})$. To study the internal structure of bands we used a high dextran concentration $(14.5 \mathrm{mg} / \mathrm{ml}$ dextran, $21.7 \mathrm{mg} / \mathrm{ml} \mathrm{fd})$. Suspensions on which experiments were performed have been prepared as follows. The homogeneous mixtures were allowed to phase separate for a few hours, after which full phase separation was achieved by gentle centrifugation $\left(10^{4} \mathrm{~g}\right.$ overnight). The binodal concentrations with added low concentration of dextran are determined to be $17.5 \pm 0.5$ and $29.0 \pm 1.5 \mathrm{mg} / \mathrm{ml}$. The width of the phase-coexistence region is considerably wider when compared to a pure fd suspension, where binodal points are at 21 and $23 \mathrm{mg} / \mathrm{ml}$, respectively. A volume $V_{\text {iso }}$ from the isotropic phase is mixed with a volume $V_{\text {nem }}$ of the coexisting nematic phase. The concentration of fd virus particles in such a mixture is expressed in terms of the quantity $\varphi_{\text {nem }} \equiv V_{\text {nem }} /\left(V_{\text {nem }}+V_{\text {iso }}\right)$, which varies from 0 for the lower binodal concentration $(17.5 \pm 0.5 \mathrm{mg} / \mathrm{ml})$ to 1 for the upper binodal concentration $(29.0 \pm 1.5 \mathrm{mg} / \mathrm{ml})$ in the absence of flow. Homogenized mixtures within the biphasic region at various concentrations $\varphi_{\text {nem }}$ are used for the vorticity-banding experiments. Such mixtures are prepared to be at constant dextran chemical potential, independent of the fd concentration. The samples with higher dextran concentration form thicker and more regular vorticity bands, and are thus more suitable to study the internal structure of the bands.

The variable $\varphi_{\text {nem }}$ defined above is not introduced here as an "order parameter," but rather as a convenient measure for the concentration of a sample relative to the two binodal concentrations. In all our experiments the actual fd concentration is always between the two binodal concentrations, so that $\varphi_{\text {nem }}$ always lies between 0 and 1 . 


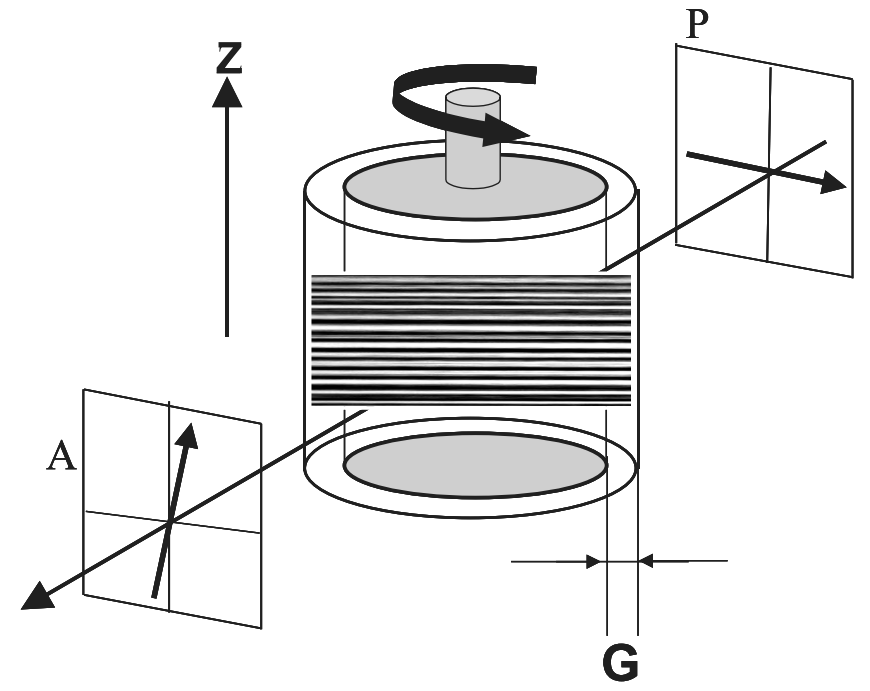

FIG. 1. Schematic of the experimental setup. The optical shear cell is placed between two polarizers, and spatial-temporal images of the banded structure are recorded with a CCD camera equipped with a telecentric lens. Additionally we show an image of a typical banded structure. The polarizers are not exactly crossed for the reasons discussed in Sec. V. The unit vector $\hat{z}$ indicates the vorticity direction.

\section{B. Experimental setup and data analysis}

Transparent couette shear cells are used with gap widths ranging from 1.0 to $2.0 \mathrm{~mm}$. The rotating inner cylinder has a radius of $24 \mathrm{~mm}$ while the outer cylinder is fixed. The experiments are performed under controlled-rate conditions. For one concentration, the determination of the border shear rates is repeated under controlled-stress conditions, using a Bohlin rheometer (CVO). The optical couette cell is placed between a polarizer and an analyzer and is illuminated from one side with a white light source, as sketched in Fig. 1. The polarizer $P$ is oriented along the flow direction. The optimal contrast between vorticity bands is obtained when the polarizer and analyzer $A$ are not exactly crossed. Typically, the angle between the analyzer and the flow direction is $80^{\circ}$. The reason for this is related to the weak rolling flow within the bands and is discussed in Sec. V. The transmitted light intensity is monitored with a 12-bit charge-coupled device CCD camera (RS Princeton Instruments) equipped with a telecentric lens (Computar $5.5 \mathrm{~mm}$ ). One pixel corresponds to $8.8 \mu \mathrm{m}$ in real space, which sets the spatial resolution in the vorticity direction of our setup. The depth of the focus is about $1 \mathrm{~mm}$. Therefore, an image is smeared along the gradient direction over a large part of the gap. Since the orientational order differs in the two types of vorticity bands, they appear in transmission as alternating bright and dark stripes, stacked along the vorticity direction (see the inset in Fig. 1).

For lower dextran concentrations, the extent of a single dark or bright band is between 50 and $120 \mu \mathrm{m}$. A single image at each time covers a region that includes about 50 vorticity bands divided over 582 pixels. The intensity at a particular height is the average over $80-100$ adjacent pixels along the flow direction. Typically, ten such cuts from a single image are analyzed as described below and averaged.
To probe the evolution of banded structures right after a shear-rate quench, optical morphologies are recorded every 10-60 s, depending on the rate of band formation, which is a function of the cell gap width, fd concentration, and shear rate. The total recording time varies from 1 to $3 \mathrm{~h}$, which is the time to reach the quasistationary banded state.

The intensity profiles are analyzed as follows. An intensity profile $I(z, t)$ at a particular time $t$ obtained from images as described above is first represented by a Fourier series as

$$
I(z, t)=I_{0}(t)+\sum_{n=1}^{N_{\max }}\left[\alpha_{n}(t) \sin (2 \pi z / L)+\beta_{n}(t) \cos (2 \pi z / L)\right],
$$

where $I_{0}$ is the average intensity, $L$ is the total height of the image, and $0 \leqslant z \leqslant L$ is the height variable along the vorticity direction. In order to avoid high-frequency peaks due to noise being identified as a vorticity band, we averaged three adjacent intensities, corresponding to $26 \mu \mathrm{m}$. The maximum number of Fourier modes $N_{\max }$ is therefore equal to one-third of the number of image pixels. A band thus encompasses at least three pixels. The average number $\bar{n}$ of bright and dark bands is now obtained from

$$
\bar{n}(t)=\sum_{n=N_{\min }}^{N_{\max }} n P_{n}(t)
$$

where $N_{\min }$ is chosen equal to 3 in order to eliminate spurious long-wavelength variations in light intensity which are much longer than a typical bandwidth, while

$$
P_{n}(t)=\frac{\sqrt{\alpha_{n}^{2}(t)+\beta_{n}^{2}(t)}}{\sum_{n=N_{\min }}^{N_{\max }} \sqrt{\alpha_{n}^{2}(t)+\beta_{n}^{2}(t)}}
$$

is the normalized probability for a Fourier mode of order $n$. The average width at a given time of a vorticity band is then obtained from

$$
H(t)=L / 2 \bar{n}(t) .
$$

As mentioned above, the choices for $N_{\max }$ and $N_{\min }$ are such that high-frequency contributions (due to noise) are eliminated and low-frequency variations (due to nonuniform illumination) do not lead to erroneous results. We confirmed by counting the number of bands by hand for a number of experiments that the procedure described above gives the correct number of bands.

\section{THE NONEQUILIBRIUM PHASE DIAGRAM}

The paranematic-nematic and vorticity-banding phase diagram in the shear rate versus fd concentration plane is presented in Fig. 2 (similar phase diagrams can be found in Refs. $[9,10])$. For fd virus suspensions, vorticity banding is observed within a part of the two-phase paranematic-nematic region which is bounded by the binodal. Dextran is added to fd rods, which induces depletion attractions. This in turn leads to a widening of the biphasic region [29] and an en- 

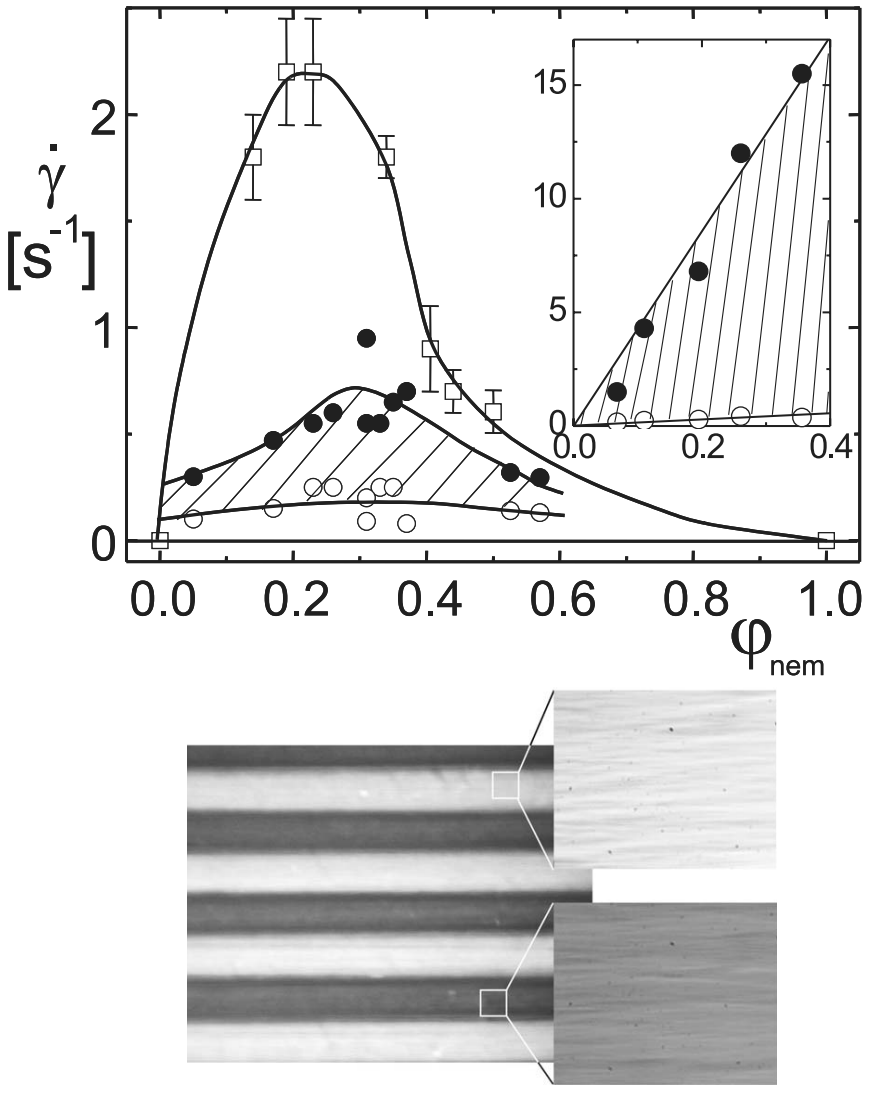

FIG. 2. The nonequilibrium phase diagram in the shear rate versus concentration plane for an overall fd concentration of $21.0 \mathrm{mg} / \mathrm{ml}$ and an overall dextran concentration of $10.6 \mathrm{mg} / \mathrm{ml}$. The fd concentration is expressed in terms of $\varphi_{\text {nem }}$ as defined in Sec. II A. The upper solid curve is the paranematic-nematic binodal and the shaded area is the region where vorticity banding is observed. The $\bigcirc$ are experimental points where banding occurs for the first time on increasing the shear rate while the are the experimental data where banding ceases to occur. The inset shows part of the vorticity-banding region for an overall dextran concentration of $14.5 \mathrm{mg} / \mathrm{ml}$. Here, the binodal is not shown. The lower figure (which is taken from Ref. [9]) shows a banded state for the same dextran concentration of $14.5 \mathrm{mg} / \mathrm{ml}$. The bandwidth is about $2 \mathrm{~mm}$. The two enlargements on the right show the inhomogeneities that are present within the bands. The thickness of these inhomogeneities is of the order of $10-20 \mu \mathrm{m}$.

hancement of both phase-separation and vorticity-banding kinetics. It is still uncertain whether banding occurs in fd suspensions without any dextran.

We first turn our attention to determining the location of the paranematic-nematic binodal under flow, which was accomplished using time-resolved shear-stress measurements after a shear-rate quench [10]. Starting at a high shear rate, where the homogeneous state is stable, the shear rate is quenched to a lower value and the shear stress is measured as a function of time. If the system crosses the binodal, inhomogeneities in concentration and orientation order develop with time, giving rise to an increase of the shear stress. On quenching from a high to a lower shear rate, isotropic inhomogeneities are formed in a nematic background. Since these isotropic inclusions have a higher viscosity as compared to the nematic background, their growth is accompanied by an increase in the shear viscosity. Such time-resolved stress measurements allow the determination of the paranematicnematic binodal in the fd concentration versus shear rate plane and lead to the upper solid curve in Fig. 2. The binodal determined in this way marks the concentrations of phase coexistence after completion of phase separation, since the homogeneous phases that coexist become metastable or unstable on increasing (for the lower binodal) or decreasing (for the upper binodal) the fd concentration. Note that this binodal is not connected to a shear-induced phase transition, contrary to many wormlike micellar systems. Here, the binodal is merely shear affected, and also occurs in the absence of flow.

The above-described method is not suitable for determining the paranematic-nematic binodal for the case of either high dextran concentration or high fd concentration. With increasing dextran concentrations the binodal is located at increasingly higher shear rates. As a result, the difference in the orientational order across the binodal is not as pronounced as for samples with lower dextran concentration. This leads to a decrease of the stress response after a shearrate quench and renders the experimental determination of the binodal difficult. For this reason we have not shown the binodal in the inset of Fig. 2. At high fd concentration $\left(\varphi_{\text {nem }}>0.7\right)$ close to the homogeneous nematic phase, states with periodic tumbling or wagging of rods in shear flow are observed $[10,41]$. This is evidenced by an oscillating shearstress response subsequent to a shear-rate quench. For homogeneous nematic phases, theory predicts such behavior for certain rod concentrations and shear rates [37,42-46]. Due to the oscillatory response, the location of the binodal as well as the extent of the vorticity-banding transition cannot be determined for $\varphi_{\text {nem }}>0.7$. Therefore, the measurements of the binodal and vorticity banding are limited to lower fd concentrations. More details about the experimental determination of the binodal are available in Ref. [10].

The vorticity-banding region is determined from profiles taken at various shear rates similar to the ones shown in Fig. 3. The profiles in Fig. 3 persist for more than a week and are taken for a fixed fd concentration $\left(\varphi_{\text {nem }}=0.23\right)$. The shear rate $\dot{\gamma}_{-}$is the lower-border shear rate of the vorticity-banding region (the lower bound of the shaded region in the phase diagram in Fig. 2), and $\dot{\gamma}_{+}$is the upper-border shear rate. As can be seen in Fig. 3, for shear rates below $\dot{\gamma}_{-}$, no clear bright and dark bands are formed. Inhomogeneities that are formed due to phase separation are stretched to a certain extent (leftmost profile in Fig. 3), but do not give rise to the formation of vorticity bands. For shear rates $\left(\dot{\gamma}_{-}<\dot{\gamma}<\dot{\gamma}_{+}\right)$ within the vorticity-banding region, clear bright and dark bands are formed (two middle profiles in Fig. 3). These profiles exhibit relatively large intensity variations and a longerwavelength structure when compared to those outside the banding region. For shear rates slightly above $\dot{\gamma}_{+}$, heterogeneous vorticity banding is observed (rightmost profile in Fig. $3)$. The shear-rate range $\left(\dot{\gamma}_{+}<\dot{\gamma}<\dot{\gamma}_{\text {het }}\right)$ where heterogeneous banding occurs is about $5-10 \%$ of the homogenous vorticity-banding shear-rate range $\left(\dot{\gamma}_{-}<\dot{\gamma}<\dot{\gamma}_{+}\right)$. Experiments of this kind are repeated for different fd concentrations 


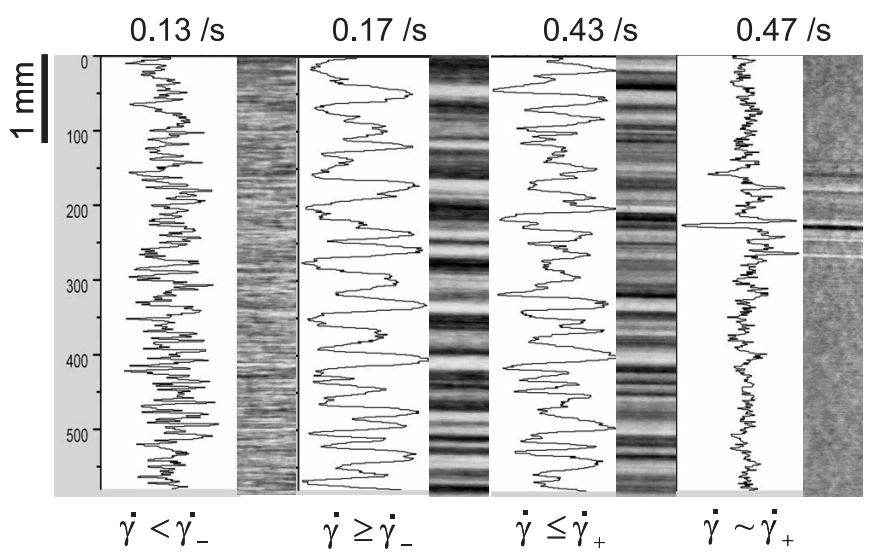

FIG. 3. Quasistationary intensity profiles taken a few hours after the shear-rate quench into the vorticity-banding region. The gap width is $2.0 \mathrm{~mm}$ and the fd concentration is $\varphi_{\text {nem }}=0.23$. The overall dextran concentration is $10.6 \mathrm{mg} / \mathrm{ml}$, which complies with the phase diagram in Fig. 2. The border shear rates $\dot{\gamma}_{-}$and $\dot{\gamma}_{+}$are the lower and higher shear rates that bound the vorticity-banding region in the phase diagram. The leftmost profile shows an image for a shear rate just below $\dot{\gamma}_{-}$. The two middle profiles are for shear rates within the banding region, while the rightmost profile is taken for the shear rate just above $\dot{\gamma}_{+}$, where heterogeneous banding occurs. The vertical axis is given in camera-pixel number and a scale bar is added on the left side. The intensity scales are the same for all four profiles.

to determine the entire vorticity-banding region in the fd concentration vs shear rate plane. For each fd concentration the final shear rate in the two-phase region is systematically varied in steps of $0.02-0.04 \mathrm{~s}^{-1}$. The prequench shear rate is always $10 \mathrm{~s}^{-1}$, which is far above the binodal.

Due to sedimentation, the system will eventually phase separate into a coexisting paranematic and a sheared nematic bulk phase. This can be seen in Fig. 4 in Ref. [9], which shows a partly demixed system where sedimentation of denser nematic inhomogeneities occurred to some extent. The upper phase in this figure is a homogeneous paranematic phase that does not contain any inhomogeneities. The absence of a banded structure in the upper phase demonstrates that the lower binodal in Fig. 2 coincides with the lower bound of the vorticity-banding region.

The inset in Fig. 2 shows the vorticity-banding region (up to $\left.\varphi_{\text {nem }}=0.4\right)$ for the higher dextran concentration of $14.5 \mathrm{mg} / \mathrm{ml}$. In this case the vorticity-banding region extends to much higher shear rates since the attractions between the rods are increased. Still, this region is contained within the biphasic region as bounded by the binodal. The vorticity bands at higher overall dextran concentrations are larger and more regular. Such bands are used for experiments on the internal flow and orientational order within the bands as described in Sec. V. The typical quasistationary banded structures that is observed for these higher dextran concentrations are shown in the lower part of Fig. 2. On the righthand side there are two microscopy images that show the inhomogeneous microstructure found within each of the bands. These inhomogeneities are due to paranematicnematic phase separation and are formed right after the
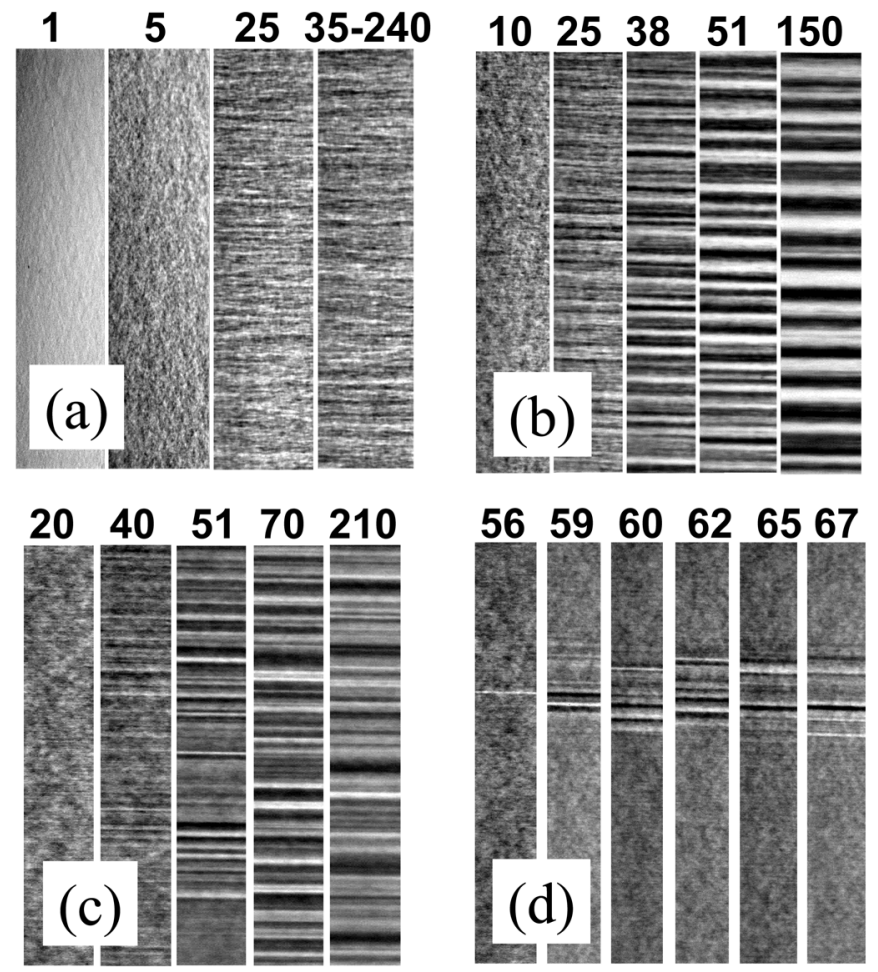

FIG. 4. Temporal evolution of the vorticity banding morphologies at various shear rates for an fd concentration of $\varphi_{\text {nem }}=0.23$ and a gap width of $2.0 \mathrm{~mm}$. The overall dextran concentration is $10.6 \mathrm{mg} / \mathrm{ml}$. The numbers above the intensity profile images refer to the time after the shear-rate quench in minutes. (a), (b) Shear rates 0.15 and $0.17 \mathrm{~s}^{-1}$ just below and above the lower-border shear rate $\dot{\gamma}_{-}=0.16 \mathrm{~s}^{-1}$, respectively. (c), (d) Shear rates 0.45 and $\dot{\gamma}_{+}$ $=0.47 \mathrm{~s}^{-1}$ just below and above the upper-border shear rate $\dot{\gamma}_{+}$ $=0.46 \mathrm{~s}^{-1}$, respectively. Heterogeneous banding is observed in (d) just above the upper-border shear rate.

shear-rate quench. In the proposed mechanism that underlies vorticity banding as presented in Sec. VI, these inhomogeneities play an essential role in the stabilization of the banded state.

\section{KINETICS OF VORTICITY BANDING}

The experiments discussed in the present section are done on fd-dextran suspensions with the lower overall dextran concentration of $10.6 \mathrm{mg} / \mathrm{ml}$. Figure 4 shows timedependent banded intensity profiles subsequent to a quench from an initial shear rate of $10 \mathrm{~s}^{-1}$ to four final shear rates located in the biphasic paranematic-nematic region. The profiles in Fig. 4(a) are for a final shear rate $0.15 \mathrm{~s}^{-1}$ just below the lower-border shear rate $\dot{\gamma}_{-}=0.16 \mathrm{~s}^{-1}$. No banding is observed. Here, the striped pattern is due to inhomogeneities that are formed due to paranematic-nematic phase separation and are elongated in shear flow. For Fig. 4(b), the final shear rate $0.17 \mathrm{~s}^{-1}$ is just above $\dot{\gamma}_{-}$. Here, the growth of the vorticity bands is clearly visible as the appearance and coarsening of bright and dark bands. Similarly, for a shear rate $0.45 \mathrm{~s}^{-1}$ just below the upper-border shear rate $\dot{\gamma}_{+}=0.46 \mathrm{~s}^{-1}$, the banding transition is clearly observed in Fig. 4(c). In a small 


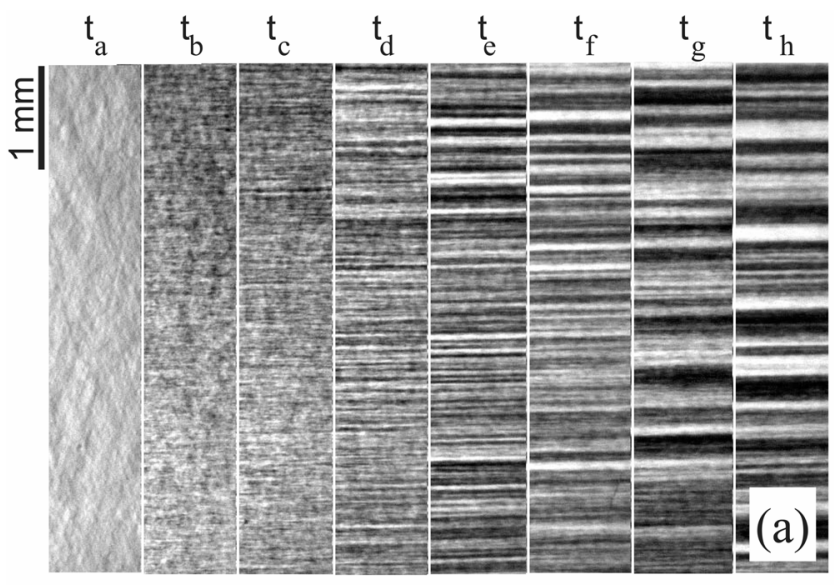

$\mathrm{H}[\mu \mathrm{m}]$

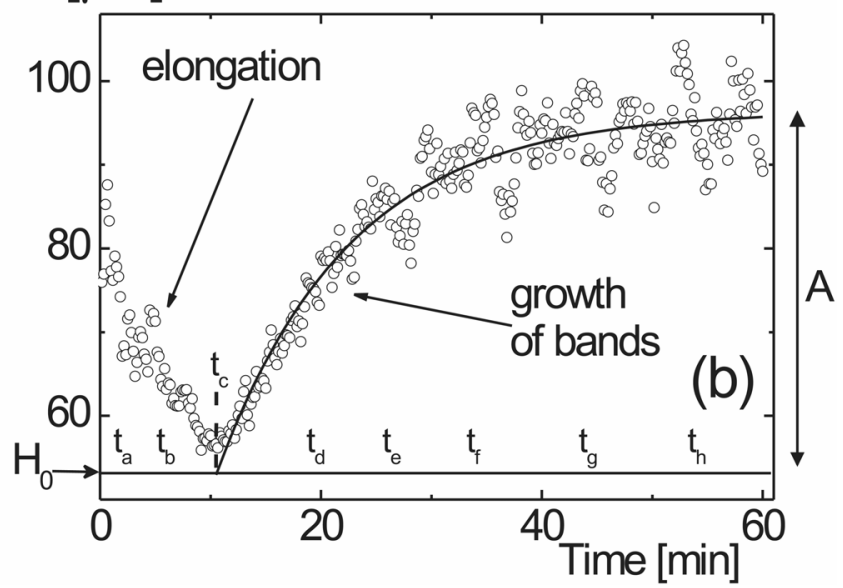

FIG. 5. (a) Temporal development of vorticity profiles right after a shear-rate quench into the banding region. (b) The bandwidth $H$ as a function of time right after a shear quench, as measured from profiles as given in (a). The solid line is an exponential fit to the data according to Eqs. (4) and (6). Here, the times $t_{a}-t_{c}$ in both figures are related to shear stretching of inhomogeneities at several times after the quench, while $t_{d}-t_{h}$ relate to growth of vorticity bands. The fd concentration is $\varphi_{\text {nem }}=0.23$, the shear-cell gap width is $2.0 \mathrm{~mm}$, and the shear rate is $0.25 \mathrm{~s}^{-1}$. The overall dextran concentration is $10.6 \mathrm{mg} / \mathrm{ml}$. The shear rate is located in the middle of the vorticity-banding region.

shear-rate region just above the upper-border shear rate $\dot{\gamma}_{+}$, heterogeneous banding occurs, as can be seen in Fig. 4(d).

In order to quantify the growth kinetics of vorticity bands, the time-dependent bandwidth is obtained from intensity profiles by the Fourier-mode analysis described in Sec. II B. A typical result of such an analysis is shown in Fig. 5(b). There is a well-defined time $t_{0}$, beyond which vorticity banding occurs. Afterward the bandwidth increases continuously until it saturates at long times (the times indicated as $t_{d}-t_{h}$ are in this regime). The time $t_{0}$ corresponds to the profile marked with $t_{c}$. From the corresponding image in Fig. 5(a) it can be seen that, indeed, at the time $t_{0}$ clear bright and dark stripes begin to appear. On average the bright and dark regions have an equal width during their growth.

It is much harder to ascertain the behavior of the system prior to the onset of the shear-banding transition. Since the depth of the camera focus is $1 \mathrm{~mm}$, the acquired image is a superposition of spatial variations of inhomogeneities along the gradient direction. Therefore, the apparent bandwidth found in this region using image analysis is highly susceptible to artifacts. The best way to study the behavior of inhomogeneities formed in this region is with small-angle light scattering (SALS). SALS data showing the evolution of coexisting droplets (inhomogeneities) upon a shear-rate quench into a biphasic region are shown in Ref. [9]. The conclusion drawn from these experiments is that right after the shearrate quench the inhomogeneities formed due to paranematicnematic phase separation are shear stretched along the flow direction. This process is usually complete in about $10 \mathrm{~min}$. After this initial stage we do not see any significant change in the SALS pattern. This indicates that any further coarsening process of coexisting phases is suppressed by the shear flow. Even after vorticity bands are fully developed it is possible to observe the presence of inhomogeneities in both bands (see lower panel of Fig. 2).

The relevant parameters obtained from growth curves like the one in Fig. 5(b) are the growth time and final bandwidth in the stationary state. These parameters are extracted as follows. We write

$$
H(t)=H_{0}+H^{\prime}(t) \text { for } t \geqslant t_{0},
$$

where $t_{0}$ is the time at which banding sets in, $H_{0}$ is the bandwidth at time $t_{0}$, and $H^{\prime}$ describes the growth of the bands. The time dependence of the vorticity-bandwidth, to within experimental error, can be described with a single time exponential,

$$
H^{\prime}(t)=A\left\{1-\exp \left[\left(t_{0}-t\right) / \tau\right]\right\} \quad\left(t \geqslant t_{0}\right),
$$

where $A$ is the total increase of the bandwidth at long times. This will be referred to as the growth amplitude while $\tau$ is the band-growth time. The final bandwidth is equal to $H_{0}$ $+A$ (see Fig. 5). The solid line in Fig. 5(b) is a best fit to Eqs. (4) and (6) for $t>t_{0}$. The parameters $A$ and $\tau$ characterize the growth kinetics of the vorticity bands.

An exponential growth of the bandwidth is observed to within experimental error. The growth time $\tau$ is therefore the relevant experimental measure for the growth rate of bands. There is as yet no theory concerning band-growth kinetics that explains exponential growth or possible deviations from exponential growth at longer times.

The growth time $\tau$ and the growth amplitude $A$ are measured as functions of shear rate and fd concentration (within the shaded region in Fig. 2). To compare such measurements for different fd concentrations, the shear rate is normalized in dimensionless units as

$$
\dot{\gamma}_{N} \equiv \frac{\dot{\gamma}-\dot{\gamma}_{-}}{\dot{\gamma}_{+}-\dot{\gamma}_{-}}
$$

Here, $\dot{\gamma}_{-}$and $\dot{\gamma}_{+}$are the lower-border and upper-border shear rates, respectively.

Systematic measurements of kinetic parameters are done for two different fd concentrations $\varphi_{\text {nem }}=0.23$ and 0.35 . The final bandwidth $H_{0}+A$ and the growth time $\tau$ are given in Figs. 6(a) and 6(b) as a function of the normalized shear rate. 

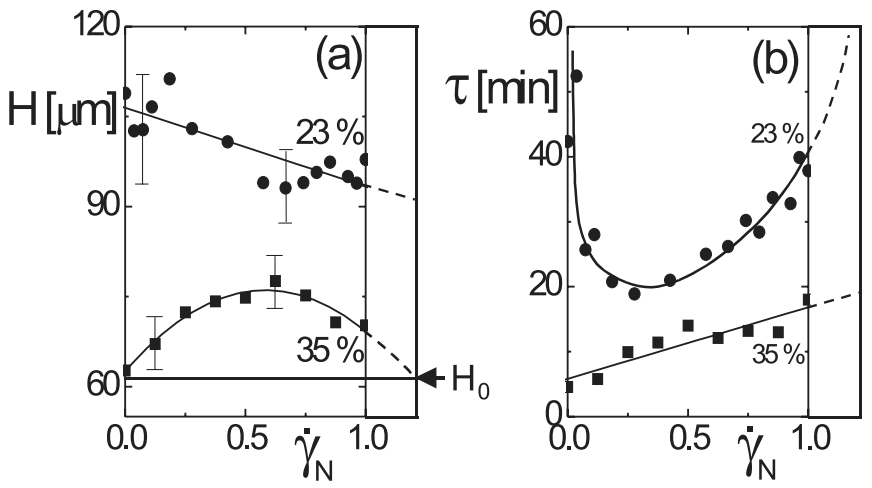

FIG. 6. (a) The final bandwidth as a function of the normalized shear rate as defined in Eq. (7) for two fd concentrations $\varphi_{\text {nem }}$ $=0.23$ and 0.35 , as indicated in the figure. In both cases the overall dextran concentration is $10.6 \mathrm{mg} / \mathrm{ml}$. (b) The band-growth time $\tau$ as a function of shear rate for the same fd concentrations. Above the upper-border shear rate $\dot{\gamma}_{+}$, heterogeneous banding occurs for both concentrations. The dotted lines are estimates of bandwidths and growth rates from intensity profiles like in Fig. 4(d).

The relatively small shear-rate range beyond $\dot{\gamma}_{+}$where heterogeneous banding occurs is also indicated in this figure. Since a Fourier-mode analysis in this region is not possible, the dashed lines are simple estimates from profiles such as the one given in Fig. 4(d).

We find an important difference in the kinetic behavior depending on the fd concentration. For both cases the bandwidth at the instant of time where banding sets in is $H_{0}$ $=60 \pm 5 \mu \mathrm{m}$. The final bandwidth is always larger than the bandwidth $H_{0}$ when banding starts. The final bandwidth for lower fd concentration $\varphi_{\text {nem }}=0.23$ varies by about $10 \%$ throughout the banding region. The band-growth time $\tau$ diverges both in the vicinity of the lower-border shear rate $\dot{\gamma}_{-}$ and below the shear rate $\dot{\gamma}_{\text {het }}$ where heterogeneous banding ceases to occur. Hence, for low fd concentration, banding ceases to occur for shear rates lower than $\dot{\gamma}_{-}$or higher than $\dot{\gamma}_{\text {het }}$ due to a vanishing growth rate $1 / \tau$. In contrast, for high fd concentration $\left(\varphi_{\text {nem }}=0.35\right)$ band-growth rates are finite throughout the banding region [lower curve in Fig. 6(b)]. The reason that banding ceases to occur in this case, for shear rates below $\dot{\gamma}_{-}$and above $\dot{\gamma}_{\text {het }}$, is that the growth amplitude $A$ vanishes [lower curve in Fig. 6(a)].

Additionally we note that the time $t_{0}$ is constant throughout the region where homogeneous banding occurs, to within experimental error. For $\varphi_{\text {nem }}=0.23, t_{0}=11 \pm 2 \mathrm{~min}$, while for $\varphi_{\text {nem }}=0.35, t_{0}=8 \pm 2 \mathrm{~min}$.

Assuming that the inhomogeneities formed due to phase separation are at the origin of the vorticity-banding instability, the different banding kinetics for the two fd concentrations are probably related to the different morphology and mechanical properties of these inhomogeneities. Figure 7 shows the morphologies for three different fd concentrations during the early stages of phase separation, obtained by confocal microscopy. These images are taken after a shear-rate quench to a zero shear rate. For the lowest concentration, an interconnected morphology is observed during the initial stages of phase separation. This is reminiscent of spinodal decomposition. After about $10 \mathrm{~min}$, the connectedness is

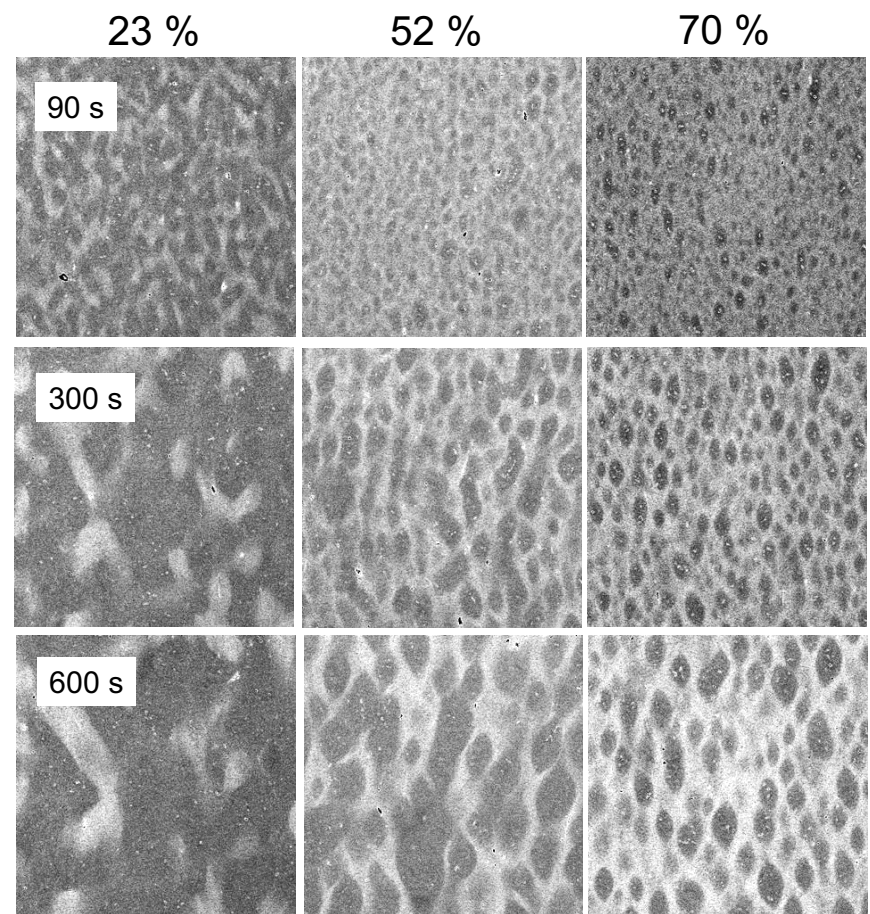

FIG. 7. Confocal images taken in reflection mode of the morphology of phase-separating suspensions, 90, 300, and $600 \mathrm{~s}$ after a shear-rate quench to zero shear rate, for three different fd concentrations, $\varphi_{\text {nem }}=0.23,0.52$, and 0.70 , as indicated in the figure. The bright regions are nematic, the dark regions are isotropic. The field of view is $180 \mu \mathrm{m}$.

lost, and a blurry morphology of inhomogeneities is formed. At the highest fd concentration, nucleation of isotropic tactoids in a nematic background is observed. A somewhat ordered, noninterconnected structure exists after about 10 min. For the middle concentration, which is close to the spinodal, interconnectedness still exists to some extent after $10 \mathrm{~min}$. Slow phase separation in the vicinity of the spinodal leads to a still interconnected structure after a relatively long time. The fact that spinodal decomposition is observed at low fd concentration and nucleation and growth at high concentration is due to the residual alignment of the fd rods subsequent to a shear quench $[47,48]$. A quantitative analysis of this type of phase-separation kinetics is given in Ref. [48], where the relevant spinodal concentration is found to be around an fd concentration of $\varphi_{\text {nem }}=0.6$. The morphologies shown in Fig. 7 will be strongly deformed under shear flow, but will probably still be very different with changing fd concentrations. Different mechanical properties of these shear-deformed inhomogeneities might be at the origin of the observed difference in the banding kinetics for the two fd concentrations. A more systematic microscopic investigation is necessary to quantify the relation between the sheardeformed morphology of inhomogeneities and the observed vorticity-banding kinetics.

As mentioned before, the spinodal and binodal referred to here are connected to the paranematic-nematic phase transition, that is, the shear-affected isotropic-nematic phase transition which also occurs in the absence of flow. It refers to the shear-affected isotropic-nematic phase transition and not to the banding transition. 

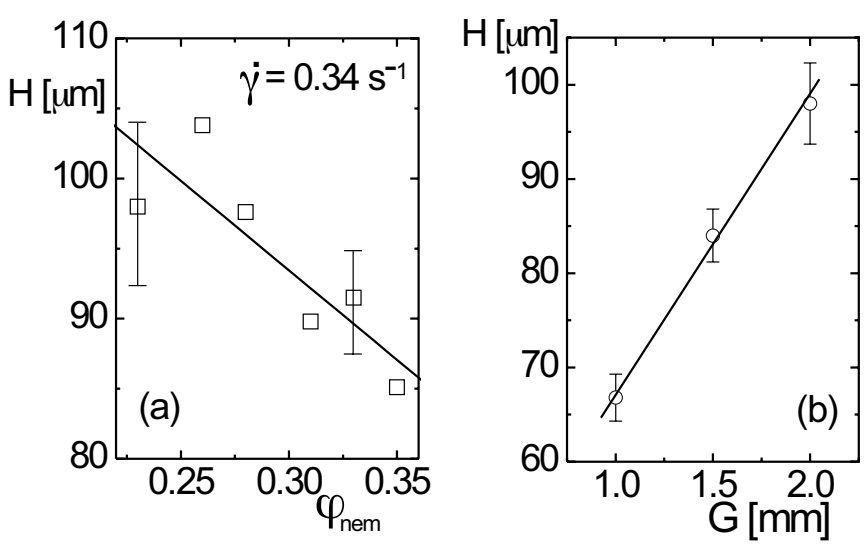

FIG. 8. (a) The concentration dependence of the final bandwidth $H$ for a shear rate equal to $0.34 \mathrm{~s}^{-1}$ and for the lower overall concentration of $10.6 \mathrm{mg} / \mathrm{ml}$. The gap width of the shear cell is $2.0 \mathrm{~mm}$. (b) The gap-width dependence of the final bandwidth for $\varphi_{\text {nem }}=0.23$. The data points are averages over shear rates within the banding region, and the error bars relate to the spread in bandwidth on variation of the shear rate [see the upper curve in Fig. 6(a)].

As will be discussed in Sec. VI, gradients in shear rate determine the normal stress that is generated by the inhomogeneities, which in turn stabilizes the quasistationary state. Since the morphology of inhomogeneities at the time that banding sets in depends on the overall fd concentration, as discussed above, the final bandwidth, therefore, might be concentration dependent. As can be seen from Fig. 8(a), the final bandwidth is indeed seen to be depending on the fd concentration. The final bandwidth decreases with increasing fd concentration. There should also be a gap-width dependence of the final bandwidth due to the fact that the gradients in shear rate increase with increasing gap width. Such a gapwidth dependence is indeed found, as shown in Fig. 8(b). As can be seen, the bandwidth increases as the gradients in shear rate become larger.

\section{STRUCTURE OF QUASISTATIONARY VORTICITY- BANDED STATE}

Two experiments provide strong indication that the vorticity banding is due to a weak rolling flow superimposed onto the applied shear flow. For both of these experiments we have used a somewhat higher dextran concentration of $12.3 \mathrm{mg} / \mathrm{ml}$ to produce large and regularly stacked vorticity bands. The width of the quasistationary bands under these conditions is about $1 \mathrm{~mm}$. In a first experiment we use polarization optics to probe the orientational order of $\mathrm{fd}$ rods within adjacent bands. The couette cell is placed between an exactly crossed polarizer and analyzer (see Fig. 1). A $\lambda / 2$ plate is placed between the couette cell and the analyzer, with a variable angle $\Theta$ with respect to the polarization direction of the analyzer. When the optical axis of the $\lambda / 2$ plate is perpendicular (or parallel) to $A$ there is no visible contrast between the two bands [middle image in Fig. 9(a)]. This implies that the orientational order averaged along the gradient direction is the same in both bands. However, all the rods along the gradient direction are not necessarily aligned

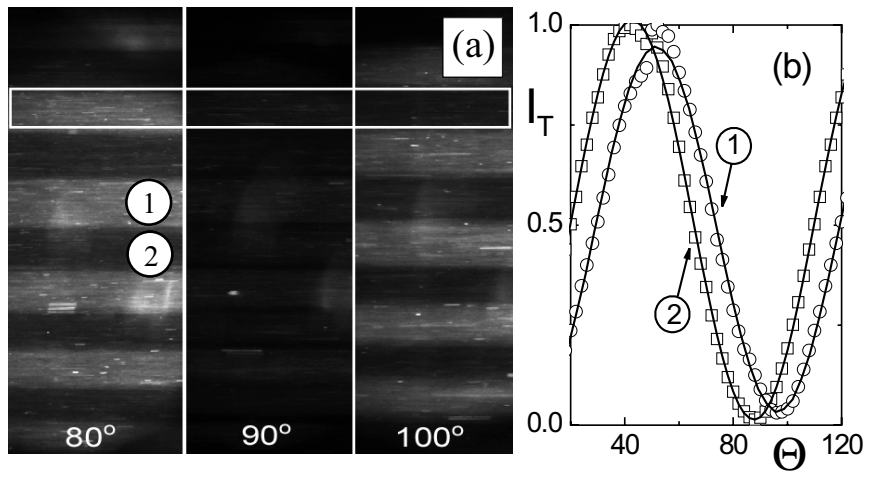

FIG. 9. (a) Images of shear bands illuminated with white light with a polarization direction parallel to the flow and the crossed analyzer directed along the vorticity direction. Between the analyzer and the couette cell there is a $(1 / 2) \lambda$ platelet with its optical axis at an angle of $80^{\circ}, 90^{\circ}$, and $100^{\circ}$ with respect to the analyzer (from left to right). The height of the images is $6.5 \mathrm{~mm}$. (b) The transmitted intensity of two adjacent bands (the bands marked as 1 and 2 as a function of the angle $\theta$. $\Theta$ is the angle between the analyzer and the $(1 / 2) \lambda$ platelet. The solid lines are best fits to a linear combination of a sine and cosine with the same period and the same offset phase. The overall dextran concentration is $12.3 \mathrm{mg} / \mathrm{ml}$, the shear rate is $0.26 \mathrm{~s}^{-1}$, and $\varphi_{\text {nem }}=0.17$.

in the same direction. In other words the rod orientation can form a left- or right-handed helix. This can be shown by changing the angle of the $\lambda / 2$ plate. The polarized light is rotated equally by the two bands but in exactly opposite directions. This is nicely demonstrated by the leftmost and rightmost images in Fig. 9(a), where the $\lambda / 2$ plate is at angles $80^{\circ}$ and $100^{\circ}$ with respect to $A$. The brightness of a band in the left image is equal to the brightness of adjacent bands in the right image. The transmitted intensity in the two bands as a function of the angle $\Theta$ is plotted in Fig. 9(b). Such behavior is consistent with banded structures that are in a rolling motion [as depicted in Fig. 10(b)]. Since the rolling motion is opposite in direction for two adjacent bands, the change of the polarization direction of light is equal but opposite in sign for the two bands. This optical phenomenon is similar to propagation of light in cholesteric liquid crystals [49].

In the second experiment focused on determining the structure of vorticity bands we have tracked tracer particles within a vorticity band. Here, a couette cell is used with two counter-rotating cylinders. The height of the cell is adjusted to keep track of the particle along the vorticity direction. The position of the tracer particle in the gradient direction is probed by changing the relative angular velocity of the two cylinders so as to shift the plane of zero velocity in order to keep the particle within the field of view. The relative angular velocities are adjusted in such a way that the average shear rate remains unchanged. As can be seen from Fig. 10 (a), the height $z$ of the tracer sphere, which is the component of its position along the vorticity direction, oscillates in time with an amplitude that is roughly equal to the bandwidth. There is drift of the $z$ position due to sedimentation of the large tracer particle. The experimental measure for the location of the tracer sphere in the gradient direction is the so-called speed ratio $S . S$ is defined as 


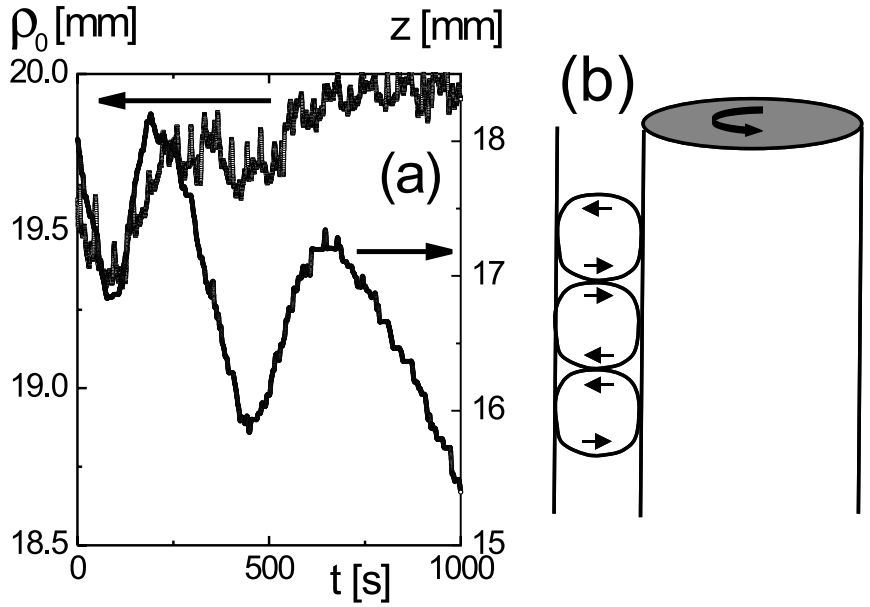

FIG. 10. (a) On the right axis, the position $z$ in the vorticity direction of a tracer sphere with a diameter of $50 \mu \mathrm{m}$ is shown as a function of time in the quasistationary banded state as measured by video microscopy with a counter-rotating couette cell, where the radii of the inner and outer cylinders are 18.5 and $20.0 \mathrm{~mm}$, respectively. The time $t=0$ is the time after which the optical trapping of the tracer sphere is released. The radial position $\rho_{0}$ of the tracer sphere is shown on the left axis. The overall dextran concentration is $12.3 \mathrm{mg} / \mathrm{ml}$, the $\mathrm{fd}$ concentration is $\varphi_{\text {nem }}=0.17$, and the shear rate is $0.88 \mathrm{~s}^{-1}$. The bandwidth is about $1 \mathrm{~mm}$. (b) A sketch of the rolling flow that complies with the observed oscillatory behavior of the position coordinate of the tracer particle.

$$
S=\frac{\Omega_{o}}{\Omega_{i}+\Omega_{o}},
$$

where $\Omega_{i}$ and $\Omega_{o}$ are the (absolute values of) the rotational velocities of the counter-rotating inner and outer cylinders, respectively. The rolling velocity is simply superimposed onto the velocity $v_{0}$ in the perpendicular direction that one would have for a Newtonian fluid in a couette cell. Indeed, heterodyne dynamic light scattering experiments within the banded state show an essentially linear velocity profile as for a Newtonian fluid. The fluid flow velocity $v_{0}$ is given as a function of the radial distance $\rho$ from the centerline of rotation as

$$
v_{0}(\rho)=\frac{\rho}{R_{o}^{2}-R_{i}^{2}}\left(\Omega_{o} R_{o}^{2}+\Omega_{i} R_{i}^{2}-\left(\Omega_{o}+\Omega_{i}\right) \frac{R_{o}^{2} R_{i}^{2}}{\rho^{2}}\right),
$$

where $R_{i}$ and $R_{o}$ are the radii of the inner and outer cylinders, respectively. The location $\rho_{0}$ of the plane of zero velocity is thus equal to

$$
\rho_{0}=R_{o} R_{i} \sqrt{\frac{\Omega_{o}+\Omega_{i}}{\Omega_{o} R_{o}^{2}+\Omega_{i} R_{i}^{2}}}=\frac{R_{o} R_{i}}{\sqrt{S R_{o}^{2}+(1-S) R_{i}^{2}}},
$$

where the definition of the experimental parameter $S$ in Eq. (8) has been substituted. This relation is used to construct the plot in Fig. 10(a) for the radial position of the tracer particle. Although there is a drift of the particle toward the outer cylinder due to gradients in the shear rate, the radial position seems to exhibit an oscillatory behavior. Since the tracer particle will migrate to the outer cylinder due to gradients in
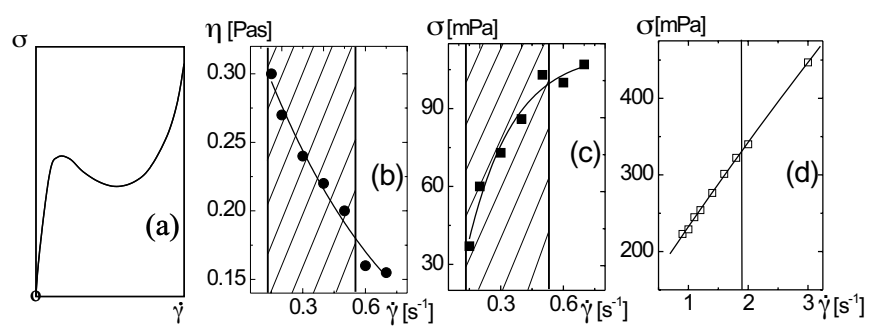

FIG. 11. (a) A typical van der Waals looplike shear-rate dependence of the shear stress for a homogeneously sheared system that will exhibit gradient banding. The viscosity $\eta$ and the shear stress $\sigma=\dot{\gamma} \eta$ as a function of shear rate are shown in (b) and (c), respectively. Vorticity banding is observed within the shaded region. The vertical bounds of the shaded area correspond to the lower- and upper-border shear rates. The data points are measured 5 min after a shear-rate quench, just before vorticity banding occurs. The fd concentration is $\varphi_{\text {nem }}=0.35$. (d) The shear stress for higher shear rates, for $\varphi_{\text {nem }}=0.33$. The overall dextran concentration is $10.6 \mathrm{mg} / \mathrm{ml}$. The vertical line indicates the location of the binodal. The gap width is $1.0 \mathrm{~mm}$.

shear rate and its initial radial position is in the middle of the gap of the shear cell, these oscillations are not very pronounced. Therefore, the data for $\rho_{0}$ are too noisy to unambiguously correlate the height and the radial position in order to prove that there is a rolling flow. The data in Fig. 10, however, strongly indicate the existence of a rolling flow, as depicted in Fig. 10(b).

Typically, $1-5 \mathrm{~h}$ are needed to establish a banded state that remains unchanged for at least a week. There is a difference between density of nematic and paranematic phase and therefore the inhomogeneities will slowly sediment over a long period of time. This will eventually lead to a state where two homogeneous bulk phases coexist: a paranematic and a sheared nematic phase (see Fig. 4 in Ref. [9].). Microscopy images and small-angle light scattering experiments indicate that inhomogeneities are present within the bands $[9,10]$. These inhomogeneities are necessary to maintain a normal stress along the gradient direction that stabilizes the vorticity bands. As soon as these inhomogeneities coalesce, for example due to sedimentation, the stabilizing normal stress ceases to exist, and bands will disappear. The banded structure is therefore a long-lived, transient state. In this sense the banded structure is referred to as quasistationary, since the inhomogeneities will not persist for ever.

\section{POSSIBLE MECHANISM FOR VORTICITY BANDING}

We have measured the flow curve of fd-dextran mixtures for the possible rheological signs of a gradient-banding transition. In the case of gradient banding, the flow curve of the homogeneous system, before banding occurs, exhibits a van der Waals looplike dependence on the shear rate. That is, there is a region of shear rates where the shear stress of the homogeneously sheared suspension decreases with increasing shear rate [see Fig. 11(a)]. A mechanism similar to gradient banding would play a role in the present system when the shear stress of the suspension just before banding sets in 
would exhibit such a decrease of the shear stress with increasing shear rate. When gradient bands are allowed to fully develop before a stress measurement is done, a plateau in the shear stress as a function of shear rate is observed under controlled shear-rate conditions [17-21]. This plateau can be tilted when the stress couples strongly to variables such as concentration [17]. There is no such signature in the flow curve for the fd suspensions within the vorticity-banding region as seen in Figs. 11(b) and 11(c). Here, the shaded area indicates the vorticity-banding region, that is, the shear-rate range $\left(\dot{\gamma}_{-}, \dot{\gamma}_{+}\right)$in the phase diagram in Fig. 2 where vorticity banding is observed. The stress and viscosity data in Figs. 11(b) and 11(c) are obtained 5 min after a shear-rate quench. Vorticity banding sets in after $10 \mathrm{~min}$. These data essentially do not change when measured within a time window of about 5-10 min after the quench. In the case of gradient banding such curves would exhibit a van der Waals looplike behavior as depicted schematically in Fig. 11(a). The system is only weakly shear thinning [see Fig. 11(b)] and does not show any sign of a van der Waals loop nor a stress plateau. In addition, we have repeated the determination of the border shear rates for one concentration under controlled stress conditions. No differences were found with the controlled rate experiments: both border shear rates are the same. The mechanism for vorticity banding is thus clearly different from that of gradient banding. Finally we confirmed that the shear stress is also well behaved throughout the entire biphasic region, as shown in Fig. 11(d). Here, the vertical line indicates the location of the binodal.

In view of the rolling flow within the bands (see Sec. V), the vorticity-banding instability might have a similar origin as the elastic instability studied in polymeric systems [35-39]. The origin of this well-known instability is as follows. When there is a gradient in shear rate, as in a couette cell, the shear-induced stretching of polymer chains leads to normal stresses along the gradient direction. Chains which are not perfectly aligned along the streamlines are stretched in a nonuniform way due to gradients in the shear rate [Fig. 12(a)]. On average, such nonuniform stretching of chains leads to normal stresses that pull a volume element toward the rotating inner cylinder [also depicted in Fig. 12(a)]. These "hoop stresses" set the fluid in motion toward the inner cylinder. This leads to a rolling flow [as sketched in Fig. 10(b)], since at the inner cylinder the flow velocity must change to the vorticity direction. In case of a free surface, the fluid may climb the inner cylinder, in which case the increase in hydrostatic pressure compensates the normal stress in the gradient direction. This is known as the Weissenberg or rodclimbing effect.

We propose that, in a similar way, nonuniform shearinduced deformation of the inhomogeneities (instead of polymer chains) may be at the origin of the vorticity-banding instability, as depicted in Fig. 12(b). Nonuniform deformation of inhomogeneities (formed during the initial stages of paranematic-nematic phase separation) due to gradients in shear rate are thus responsible for the vorticity-banding instability and the stabilization of the banded structures.

There are, however, additional forces that might play a role here. Since inhomogeneities are very much extended along streamlines, bending elasticity might give rise to sig-
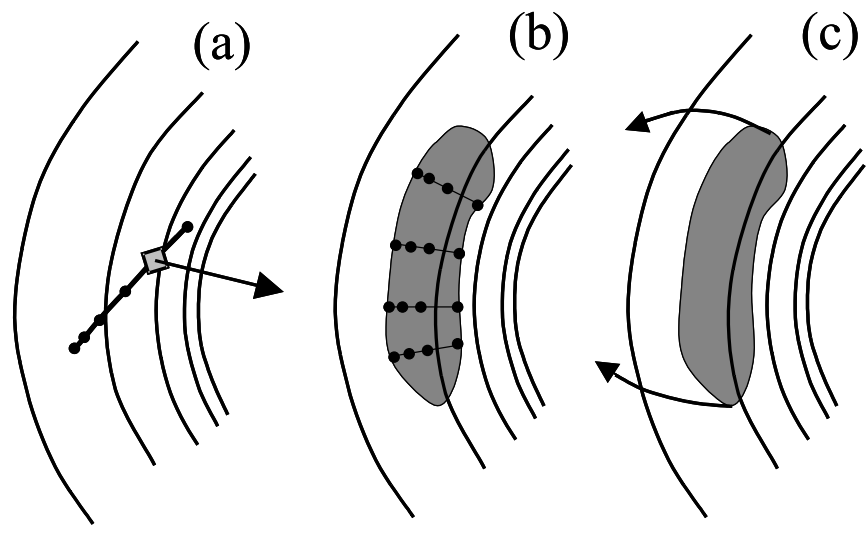

FIG. 12. (a) The origin of the well-known elastic instability of polymer systems. The dots on the polymer chain are used to indicate the degree of stretching. Without stretching or for uniform stretching, the dots would be equally spaced. When a chain is not aligned along streamlines, stretching is nonuniform and normal stresses are generated. The resulting forces pull a volume element toward the rotating inner cylinder, as indicated by the arrow. (b) Similar stretching of the inhomogeneities that are formed due to nonequilibrium paranematic-nematic phase separation. The dots are used to visualize nonuniform stretching. (c) In comparison to flexible polymer chains, bending elasticity may play a role as well. In such cases normal stresses act in the opposite direction, as depicted by the arrows in the figure.

nificant normal stresses in the opposite direction, away from the center of the couette cell [see Fig. 12(c)]. Bending elasticity [49] might counteract the stretching forces in generating hoop stresses.

The following experimental observations can be intuitively understood on the basis of the above-proposed mechanism.

(i) At a given overall shear rate, gradients in the shear rate in a couette cell increase with increasing gap width of the couette cell. Therefore the driving force for rolling flow will increase with increasing gap width. This will probably lead to an increase of the bandwidth. Indeed an increase of the bandwidth with increasing gap width is observed [see Fig. 8(b)].

(ii) Shear gradients in a couette cell are large when the overall shear rate is large. This might explain why vorticity banding occurs only at sufficiently high shear rates, that is, the lower-border shear rate $\dot{\gamma}_{-}$is larger than zero. Gradients in shear rate are not sufficiently pronounced for shear rates below $\dot{\gamma}_{-}$to render the normal stresses strong enough to induce vorticity banding.

(iii) At larger shear rates, close to the upper-border shear rate, inhomogeneities are relatively thin due to shear stretching. This diminishes the nonuniform stretching within the inhomogeneity [see Fig. 12(b), where now the inhomogeneity is very thin]. In addition, the bending forces will be smaller, since a thinner inhomogeneity is more easily bent as compared to a thick inhomogeneity. This might explain why banding ceases to occur above the upper-border shear rate $\dot{\gamma}_{+}$, which is well within the two-phase region.

(iv) Contrary to gradient banding, the region where vorticity banding occurs and the final bandwidth are indepen- 
dent of whether the shear rate or shear stress is controlled. This agrees with the mechanism proposed above, since normal stresses are important instead of shear-gradient stresses.

Although the mechanism proposed above is in accord with a number of experimental observations, more theoretical and experimental work is needed to validate these suggestions. Measurements of normal stresses along the gradient direction during the initial stages of banding could be performed. This will not be straightforward since, in weak rolling motion, these stresses are probably quite small. In addition, a detailed experimental study of the dependence of the flow pattern within the bands on varying gap width would be valuable.

Stationary vorticity-banded states are also found in aggregated nanotube suspensions [50]. Instead of the inhomogeneities formed due to phase separation as in our fd virus suspensions, here, similar deformation of the nanorod aggregates may be at the origin of the vorticity banding. Normal stress measurements reported in Ref. [50] indeed indicate that such stresses play a role in vorticity banding. Stationary vorticity banding has also been observed in wormlike micellar systems [23]. There are two possibilities here: either inhomogeneities are formed due to a shear-induced paranematic-nematic phase transition, or the wormlike micelles themselves are nonlinearly stretched (like polymer chains in the elastic instability of polymer systems). In those wormlike micellar systems where a shear-induced paranematic-nematic phase transition occurs and no vorticity banding is observed, the mechanical properties related to stretching of the inhomogeneities are probably such that normal stresses are not large enough to give rise to rolling flow. In the weakly flocculating systems in Ref. [22], the nonuniform stretching of flocs of colloidal particles is probably at the origin of the observed vorticity banding.

\section{SUMMARY}

We have performed experiments under steady shear-flow conditions on the structure and kinetics of vorticity banding in sheared suspensions of rodlike fd virus. We determined the vorticity- banding region which is entirely enclosed by the paranematic-nematic binodal. Under both controlled shear-rate and shear-stress conditions banding occurs between the lower- and upper-border shear rates $\dot{\gamma}_{-}$and $\dot{\gamma}_{+}$, respectively, where $\dot{\gamma}_{-}$is larger than zero. After a shear-rate quench from a high shear rate into this region, inhomogeneities are formed due to phase separation. These inhomogeneities are shear elongated up to a well-defined time after which vorticity banding occurs. The growth of the vorticitybandwidth can be described, to within experimental error, by a single-exponential function of time. There are two important parameters that characterize the kinetics of band formation: (i) the band-growth time $\tau$ in the exponential, the inverse of which measures the growth rate of band formation, and (ii) the amplitude $A$ of the time exponential, which is related to the total growth of the bandwidth as compared to the initial apparent bandwidth of shear-stretched inhomogeneities. The growth kinetics depends on the morphology and the mechanical properties of the inhomogeneities formed due to the phase separation. Two different scenarios have been found. For small fd concentration, $\tau$ diverges at the border shear rates, while the amplitude $A$ remains finite. The growth rate of bands thus vanishes. For larger fd concentration, the amplitude $A$ vanishes at the border shear rates, while $\tau$ remains finite.

Experiments indicate that there is a weak rolling flow within the bands. A possible mechanism that is at the origin of the banding instability as well as the stabilization of quasistationary banded states is proposed, where the mechanical properties of the inhomogeneities are essential. The proposed mechanism is similar to the elastic instability for polymer systems, where the inhomogeneities play the role of the polymer chains.

The proposed mechanism explains, on an intuitive level, a number of the observed phenomena, like the gap-width dependence of the vorticity-bandwidth, the large bandwidth with increasing dextran concentration, and the fact that banding occurs only beyond a certain nonzero shear rate and ceases to occur above another shear rate. Moreover, the proposed mechanism explains why the border shear rates are independent of whether controlled shear rates or controlled shear stresses are applied. Just above the upper-border shear rate there is a finite probability to have a localized assembly of neighboring inhomogeneities which are still thick enough to give rise to banding. This leads to the observed heterogeneous band formation.

More experiments are necessary to unambiguously validate the proposed mechanism. In addition, theory should be developed to confirm such a scenario. In particular, the expression for the stress tensor as obtained in Ref. [51], which is valid for highly inhomogeneous systems of stiff, long, and thin rods, could serve as a starting point for the theoretical validation of the proposed mechanism. Simulations also might provide a better understanding of the behavior of colloidal rods under shear flow $[52,53]$.

\section{ACKNOWLEDGMENTS}

This work was performed within the framework of the Transregio SFB TR6 "Physics of Colloidal Dispersions in External fields," and is also part of the research being performed within the European Network of Excellence "Soft Matter Composites" (SoftComp). Z.D. is supported by the Rowland Institute at Harvard. The confocal microscopy experiments have been done in cooperation with D. Derks, A. Imhof, and A. van Blaaderen, from the Utrecht University in The Netherlands. The tracking experiment has been done in cooperation with M. Lenoble and B. Pouligny from the Centre Recherche Paul Pascal, CNRS, Pesac in France. 
[1] W. C. K. Poon and P. N. Pusey, in Observation, Prediction, and Simulation of Phase Transitions in Complex Fluids, edited by M. Baus, L. F. Rull, and J. P. Ryckaert (Kluwer Academic Publishers, Dordrecht, 1995).

[2] G. J. Vroege and H. N. W. Lekkerkerker, Rep. Prog. Phys. 8, 1241 (1992).

[3] A. Baranyai and P. T. Cummings, Phys. Rev. E 60, 5522 (1999).

[4] S. Butler and P. Harrowell, Nature (London) 415, 1008 (2002).

[5] M. C. Cross and P. C. Hohenberg, Rev. Mod. Phys. 65, 851 (1993).

[6] J. F. Berret, Langmuir 13, 2277 (1997).

[7] P. Boltenhagen, Y. Hu, E. F. Matthys, and D. J. Pine, Phys. Rev. Lett. 79, 2359 (1997).

[8] T. A. J. Lenstra, Z. Dogic, and J. K. G. Dhont, J. Chem. Phys. 114, 10151 (2001).

[9] J. K. G. Dhont, M. P. Lettinga, Z. Dogic, T. A. J. Lenstra, H. Wang, S. Rathgeber, P. Carletto, L. Willner, H. Frielinghaus, and P. Lindner, Faraday Discuss. 123, 157 (2003).

[10] M. P. Lettinga and J. K. G. Dhont, J. Phys.: Condens. Matter 16, S3929 (2004).

[11] J.-F. Berret, Langmuir 13, 2227 (1997).

[12] E. Fischer and P. T. Callaghan, Phys. Rev. E 64, 011501 (2001)

[13] C. Pujolle-Robic and L. Noirez, Nature (London) 409, 167 (2001).

[14] P. T. Mather, A. Romo-Uribe, C. D. Han, and S. S. Kim, Macromolecules 30, 7977 (1997).

[15] N. Grizzuti and P. L. Maffettone, J. Chem. Phys. 118, 5195 (2003).

[16] S. Lerouge, J. P. Decruppe, and C. Humbert, Phys. Rev. Lett. 81, 5457 (1998).

[17] P. D. Olmsted and C.-Y. David Lu, Phys. Rev. E 60, 4397 (1999).

[18] C.-Y. David Lu, P. D. Olmsted, and R. C. Ball, Phys. Rev. Lett. 84, 642 (2000)

[19] P. D. Olmsted, O. Radulescu, and C.-Y. Lu, J. Rheol. 44, 257 (2000).

[20] J. L. Goveas and P. D. Olmsted, Eur. Phys. J. E 6, 79 (2001).

[21] J. K. G. Dhont, Phys. Rev. E 60, 4534 (1999).

[22] J. Vermant, L. Raynaud, J. Mewis, B. Ernst, and G. G. Fuller, J. Colloid Interface Sci. 211, 221 (1999).

[23] D. Bonn, J. Meunier, O. Greffier, A. Al-Kahwaji, and H. Kellay, Phys. Rev. E 58, 2115 (1998).

[24] P. Fischer, E. K. Wheeler, and G. G. Fuller, Rheol. Acta 41, 35 (2002).

[25] L. Onsager, Ann. N.Y. Acad. Sci. 51, 62 (1949).

[26] Z. Dogic and S. Fraden, in Soft Condensed Matter, edited by G. Gompper and M. Schick (Viley-VCH, Weinheim, 2006).
[27] J. Tang and S. Fraden, Liq. Cryst. 19, 459 (1995).

[28] E. Grelet and S. Fraden, Phys. Rev. Lett. 90, 198302 (2003).

[29] Z. Dogic, K. R. Purdy, E. Grelet, M. Adams, and S. Fraden, Phys. Rev. E 69, 051702 (2004).

[30] F. Livolant, Physica A 176, 117 (1991).

[31] S. Fraden, G. Maret, D. L. D. Caspar, and R. B. Meyer, Phys. Rev. Lett. 63, 2068 (1989).

[32] M. P. B. van Bruggen, J. K. G. Dhont, and H. N. W. Lekkerkerker, Macromolecules 32, 7037 (1999).

[33] J. Tang and S. Fraden, Phys. Rev. Lett. 71, 3509 (1993).

[34] J.-F. Berret, D. C. Roux, and G. Porte, J. Phys. II 4, 1261 (1994).

[35] K. Weissenberg, Nature (London) 159, 310 (1947).

[36] C. W. Macosko, Rheology, Principles, Measurements and Applications (VCH Publishers, Cambridge, U.K., 1994).

[37] R. G. Larson, S. J. Muller, and E. S. G. Shaqfeh, J. Fluid Mech. 218, 573 (1990).

[38] P. Pakdel and G. H. McKinley, Phys. Rev. Lett. 77, 2459 (1996).

[39] A. Groisman and V. Steinberg, Phys. Fluids 10, 2451 (1998).

[40] T. Maniatis, J. Sambrook, and E. F. Fritsch, Molecular Cloning: A Laboratory Manual, 2nd ed. (Cold Spring Harbor Laboratory Press, Plainview, NY, 1989).

[41] M. P. Lettinga, Z. Dogic, H. Wang, and J. Vermant, Langmuir 21, 8048 (2005)

[42] S. Hess, Z. Naturforsch. A 31, 1034 (1976).

[43] G. Rienäcker, M. Kröger, and S. Hess, Phys. Rev. E 66, 040702(R) (2002).

[44] S. Hess and M. Kröger, J. Phys.: Condens. Matter 16, S3835 (2004).

[45] M. G. Forest, Q. Wang, and R. Zhou, Rheol. Acta 44, 80 (2004).

[46] M. G. Forest, X. Zheng, R. Zhou, Q. Wang, and R. Lipton, Adv. Funct. Mater. 15, 2029 (2005).

[47] J. K. G. Dhont and W. J. Briels, Phys. Rev. E 72, 031404 (2005).

[48] M. P. Lettinga, K. Kang, A. Imhof, D. Derks, and J. K. G. Dhont, J. Phys.: Condens. Matter 17, S3609 (2005).

[49] P. G. de Gennes and J. Prost, The Physics of Liquid Crystals, 2nd ed. (Clarendon Press, Oxford, 1999).

[50] S. Lin-Gibson, J. A. Pathak, E. A. Grulke, H. Wang, and E. K. Hobbie, Phys. Rev. Lett. 92, 048302 (2004).

[51] J. K. G. Dhont and W. J. Briels, J. Chem. Phys. 117, 3992 (2002); 118, 1466 (2003).

[52] R. Zhou, M. G. Forest, and Q. Wang, Multiscale Model. Simul. 3, 853 (2005).

[53] G. Germano and F. Schmid, J. Chem. Phys. 123, 214703 (2005). 